\section{Open data: enforce materials sharing}

The deposition and sharing of research materials is an often-overlooked but crucial aspect of the debate on open data, even though deposition is straightforward and free of charge (see Nature 546, 327; 2017). Although Nature, for instance, has a clear policy on materials sharing and deposition (see go.nature.com/2tj3hv5), sending a materials request to a busy lab can incur delays or be unproductive if, say, the first author has moved on. Moreover, good reagents will be requested hundreds of times, but few labs want to process that many requests.

Centralized repositories such as Addgene, a non-profit global repository that archives and distributes plasmids for scientists (www.addgene.org), are equipped to handle the widespread sharing of materials. Such repositories stand to accelerate science by helping the community to make the most of useful reagents and promote scientific reproducibility.

Materials sharing is also good for the impact of both scientists and journals. For example, papers for which authors have deposited a plasmid with Addgene receive substantially more citations than those that have not (see go.nature. com/2u7ecyo).

Joanne Kamens Addgene, Cambridge, Massachusetts, USA. joanne.kamens@addgene.org Competing financial interests declared (seego.nature.com/2vrjygt).

\section{Open data: support from Swiss funder}

You err in citing the Swiss National Science Foundation (SNSF) as a funding agency that forbids its grant-holders to use commercial data platforms such as figshare (Nature 546, 327 ; 2017). The SNSF strongly supports the sharing of data and open access to data, for the very reasons you mention.

The SNSF asks investigators to submit a data-management plan with their research proposals. Grant-holders may deposit their scientific data in any recognized digital archive (commercial or not) that meets the FAIR principles: Findability, Accessibility, Interoperability and Reusability (M. D. Wilkinson et al. Scientific Data 3, 160018; 2016). We refer applicants to the registry of recognized research-data repositories at www.re3data.org, which includes figshare.

We agree that the true costs of data openness must not be overlooked, which is why we routinely provide successful applicants with up to 10,000 Swiss francs (US\$10,500) to cover these costs. More funds are available if applicants can justify the required budget in detail.

We are glad that Nature supports efforts such as ours to promote open access to data, by enforcing clear data standards and the use of repositories as a condition of publication.

Matthias Egger, Angelika

Kalt Swiss National Science Foundation, Bern, Switzerland. matthias.egger@snf.ch

\section{Factor in species' conservation value}

I agree that we need to consider the diversity of species' functions, particularly those that are crucial for maintaining ecosystems (Nature 546, 22-24; 2017). However, functional diversity alone is not enough if we are to reach global conservation targets.

First, we have little idea of the functional traits of most insect species - by far the most speciesrich taxonomic group. Second, we need to define what makes up a 'healthy' ecosystem (for example, it might simply reflect nature's value to humans). Third, we must not sacrifice biodiversity for functionality.
A degraded ecosystem can still have high functional value (see Nature 546, 7-8; 2017), as can a community of alien invasive species. Your example of three barnacles that may be functionally less useful than the trio of a starfish, an anemone and a seagrass is correct. But we might come to a different conclusion if one of the barnacles is threatened with extinction and the starfish and anemone are invasive.

We therefore need to include 'conservation value' as a fourth stool leg, in addition to functional, phylogenetic and species diversity. In my view, it is the most important one.

Axel Hochkirch Trier University, Germany.

hochkirch@uni-trier.de

\section{Alternative telescope site is a good back-up}

Your report on discussions about a possible location for the Thirty Meter Telescope (TMT) calls for some clarification (Nature http://doi.org/b9px (2017); see also Nature http://doi.org/ b9pz; 2016). Although the TMT board's preferred site is Mauna Kea in Hawaii, the alternative location at the Observatorio del Roque de los Muchachos (ORM) on La Palma, Canary Islands, is not as disastrous as you imply.

Last year's evaluation by the TMT committee revealed that turbulence and average dust at ORM are similar to those at Mauna Kea (see go.nature. com/2ut8vfs). Indeed, dust densities above 100 micrograms per cubic metre, when the telescope's enclosure must be shut, are statistically more frequent at Mauna Kea. The fraction of clear nights is therefore about the same for both sites.

The committee concluded that ORM is an excellent alternative to Mauna Kea for operating a telescope that has to use adaptive optics. The only observations affected are those of the thermal infrared, which can be improved by flexible scheduling.

In addition, the atmospheric stability is better in La Palma than at the European Southern Observatory's Extremely Large Telescope on Cerro Armazones, Chile - the TMT's leading competitor. Because the heights of the locations are similar, the disadvantages in the thermal infrared are nearly the same relative to Hawaii.

Sergio Bonaque-González Wooptix, San Cristobal de La Laguna, Tenerife, Spain. sergiob@wooptix.com

\section{History's take on the atomic forge}

Sergei Kalinin and colleagues discuss the latest developments in manipulating matter at the nanoscale, using beams of electrons in aberrationcorrected scanning transmission electron microscopes (STEMs) (Nature 539, 485-487; 2016). Such investigations have a long history of success, hidden in the hexagonal image of uranium atoms portrayed in their article.

The STEM micrograph shown is a colorized version of one taken by my group more than three decades ago (see M. Isaacson, M. Utlaut and M. Ohtsuki in Introduction to Analytical Electron Microscopy (eds J. J. Hren et al.); Springer, 1979). It was later displayed on the cover of R. H. Petrucci's General Chemistry textbook in 1982, with appropriate credits.

Probes with 0.5 -nanometre resolution were developed in the 1970 s and soon used to fabricate nanometre-scale structures in STEM (see, for example, A. Muray et al. Appl. Phys. Lett. 45, 589-591; 1984). The advent of aberration-corrected STEMs in the past decade and of probes of 0.1-nm diameter now allows us to push the limits of imaging and fabrication to the ångström level.

Michael Isaacson University of California at Santa Cruz, USA. msi@soe.ucsc.edu 